\title{
Computer Game for Small Pets and Humans
}

\author{
Roger Thomas Kok Chuen Tan, Adrian David Cheok, \\ Roshan Lalintha Peiris, I.J.P. Wijesena, \\ Derek Bing Siang Tan, Karthik Raveendran, \\ Khanh Dung Thi Nguyen, Yin Ping Sen, and Elvin Zhiwen Yio \\ Mixed Reality Lab and Department of Biological Sciences, \\ National University of Singapore, \\ metazoa@mixedrealitylab.org \\ wWw.mixedrealitylab.org
}

\begin{abstract}
Interactive media not only should enhance human-to-human communication, but also human-to-animal communication. We promote a new type of media interaction allowing human users to interact and play with their small pets (like hamsters) remotely via Internet through a mixed-reality-based game system "Metazoa Ludens". To examine the systems effectiveness: Firstly, the positive effects to the hamsters are established using Body Condition Score study. Secondly, the method of Duncan is used to assess the strength of preference of the hamsters towards Metazoa Ludens. Lastly, the effectiveness of this remote interaction, with respect to the human users as an interactive gaming system with their pet hamsters, is examined based on Csikszentmihalyi's Flow theory [1. Results of these studies have shown positive remote interaction between human user and their pet friends. This paper provides specific experimental results on the implemented research system, and a framework for human-to-animal interactive media.
\end{abstract}

Keywords: Human-animal interaction, mixed reality, multimodal interaction, computer gaming, communication.

\section{Introduction}

With the advancement of technology, longer working hours are expected in the professional world to match up with the higher level of efficiency [2]. Due to this change in the professional lifestyle [3] humans are out of the house for longer hours and pets are often neglected and taken for granted by their owners. With this negligence, pets will be deprived of love and care [4] as well as a good dose of exercise with suitable diet to ensure healthy living [5]. Thus there is a need to create an interface that is capable of allowing humans to shower their pets with attention locally or remotely; the element of exercise may be incorporated into the system to add yet another beneficial feature for small pets.

This new interface could give a different form of gameplay and connectivity between humans and small animals. In a mixed reality gameplay, different forms of interaction may be introduced where the small animals are allowed to "chase

L. Ma, R. Nakatsu, and M. Rauterberg (Eds.): ICEC 2007, LNCS 4740, pp. 28-38, 2007.

(C) IFIP International Federation for Information Processing 2007 
after" the human owners in a digital world (which is impossible in the physical world). Such gameplay may be extended to remote interaction over the Internet. Metazoa Ludens is therefore devised.

\subsection{Objectives}

Metazoa Ludens is a system that enables humans to play games with small animals in a mixed reality environment. This human-pet computer game system illustrates a way to reverse the trend of the increased lack of quality time between humans and pets. It creates a media interface capable of remote human-animal interaction and the way they may interact with the interface. The system will provide a way for human to interact remotely with their smaller pets and also provide health benefits to the pets.

In certain parts of the world, like Asia, where living spaces are smaller and larger animals like cats and dogs are not viable to be kept as pets, smaller animals like hamsters and fishes become a more popular choice. Therefore, smaller animals are the target pets for our research system Metazoa Ludens. For Metazoa Ludens, hamsters are specifically chosen for the following advantages:

- Domesticated hamsters' running spaces are normally within their cages. Therefore we would like to encourage a new media which will allow more variety of in-house-play for hamsters.

- Hamsters are a popular pet choice and the most popular of the smaller rodents [6]. They are economical and easy maintenance pets, kept by males and females as well as the rich and poor [7]. Therefore we can create a media which can be enjoyed by a wide range of society.

- Hamsters are naturally skillful intelligent runners, which is very suitable for fun gameplay with humans 8 .

- Hamsters' natural habitat are burrows that have many tunnels and their natural behavior is to tunnel [6]. This feature is used to promote their attractive pleasure in the game play of our system.

- Hamsters have cheek pouches to store food. This is convenient for both humans and pets as they can to collect their reward from the attractor in our system [6].

It is noted that such an interface is meant to enhance human-animal interaction by allowing humans to continue interacting with their pet hamsters even in a remote situation, and is not meant to replace conventional human-animal interaction such as touch and hugging. A study showing that Metazoa Ludens system is beneficial to the hamsters based on Body Condition Scoring study (refer to section 4.1). In addition, a user survey is carried out to evaluate Metazoa Ludens system as a game by breaking down the system using features as described by Csikszentmihalyi's Flow theory 1]. The reason for using this theory for our experimental testing of our human-animal play system is substantiated by the academic GameFlow model [10] which states the appropriateness of using Flow theory to assess not just optimal performance condition but using it to assess the user's enjoyment of a game; the game in our case would be our 
system Metazoa Ludens. These studies were carried out based on these strong, dependent theoretical models as mentioned above to assess Metazoa Ludens in terms of the positive benefits to the hamsters and as an enjoyable interface to the human owners.

\section{Related Works}

Most interactions between pet owners and their pets typically involve simple games such as fetching objects or chasing squeaky toys. A more established tool-based interaction used for training dogs known as click training [1] uses a click sound as a signal before a treat is given to the pet. Augmenting these games with sophisticated technology (such as those seen in video game systems) could enrich the human-animal interaction experience. Poultry Internet [3] is a remote human-pet interaction system that allows owners to pet a chicken over the internet when they are away from home. Similarly, Petting Zoo [12] and Cat Toy [13] enable the pet owner to take care of their pet by monitoring it over the internet through a webcam. SNIF [15] is a system based on the concept of 'petworking' where owners can also learn about other animals (and their owners) that interact with their dog. Infiltrate 14 takes a different approach by displaying a virtual scene from the perspective of a fish in a fish tank. However, these systems only provide a one-way interaction with no means for the animals to directly interact with the human. Recently, Netband [16] is developing an internet based system for rearing a chick. Owners can tend to the pet by feeding them and cleaning them via tele robotic and sensor apparatus. This system purely functions as a remote means of rearing a chick and does not allow the owner to interact with the chick in an intimate manner. Thus, there is a need to create a system that is capable of enriching the interactivity between living animals and their owners by utilizing the power of advanced interactive media technologies. It is also important to note that owners spend many hours at work or on business trips, away from home and their pets. Rover@Home [11] is a system that uses common internet communication tools such as webcams, microphones and speakers to communicate with dogs over the internet. Therefore, there is a need to incorporate remote communication technology into a system to allow remote interactions between humans and their pets.

Mixed reality games allow remote/internet players to interact with each other in a more physical and ubiquitous manner. In Cricket-Controlled Pacman [18], a human player can play the traditional computer game of Pacman with real live crickets in a variant of the standard mixed reality game. The crickets act as the ghosts and run in a real maze, while the human plays the part of the Pacman and controls his virtual character on a game screen. To make the game more enjoyable for humans, the crickets are constantly kept moving by vibrating the ground in the real maze with the help of motors. A negative motivation (fear from the vibration) is employed to ensure that the crickets appear to be chasing the human.

Metazoa Ludens extends previous human-animal interactive systems by allowing bidirectional interaction between humans and their pet hamsters via a 


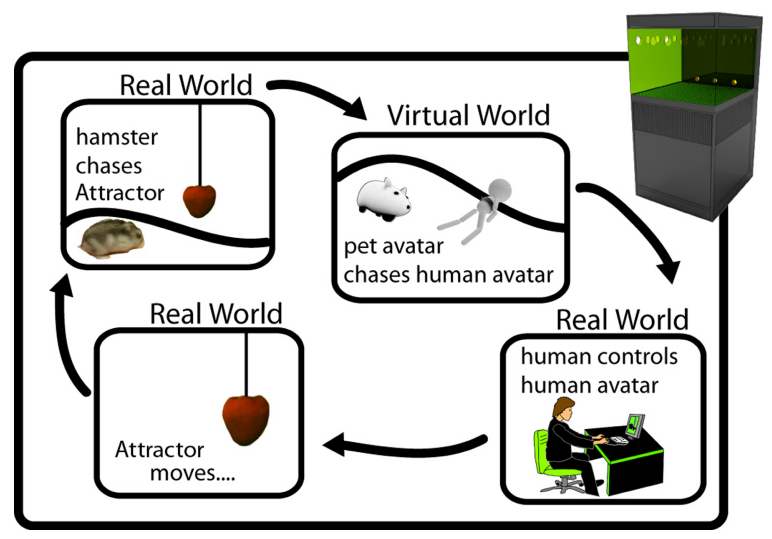

Fig. 1. System Overview

computer game. It should be noted that Metazoa Ludens is an interface meant for the benefit of small animals such as hamsters, as well as for the promotion of awareness of human-animal interaction. Positive motivation is employed so that hamsters play the game willingly and not out of fear or agitation. In addition, the game play ensures health exercise for the hamsters, which is beneficial for the well being of the pets.

\section{Game Mechanics and Gameplay}

Inside the structure a camera is placed at the top of the structure for tracking of the hamsters' movements on a mechanical driven arm that makes up the movable attractor. The surface which the pet hamsters scamper upon is a highly moldable latex sheet molded by sixteen actuators (electric motor driven) placed directly beneath them. Technical details regarding the subsystem are describe in previous works 19 .

At the start of the game the pet owner moves a human avatar across the changing virtual terrain. This controls the mechanical attractor within the tank which then moves in correspondence to the human avatar. The latex surface changes in surface contour as molded by the actuators below, this in turn corresponds to the changing contours of the virtual terrain accordingly.

The hamsters chase after the mechanical attractor. The hamsters movements are tracked by the cameras at the top of the structure, mapping its positions and moving the pet avatar in the virtual world.

The basic game concept behind Metazoa Ludens game is a predator and prey chase game. However roles are reversed. The bigger human is now being chased by the physically small hamsters in the virtual world (pet avatar chases after human avatar). A general strategy of the pet owner is to try her best to evade the pet avatar (hamster). Getting caught will then deplete the human avatar's health points. As the game progresses the human avatar will have to evade the 
pet avatar within a certain time without depleting all her health. The game does not try to replace conventional human-pet interaction, but with the aid of a digital system it adds more variety to the way a pet owner may play with their small pets, especially remotely.

\section{Evaluation, Results and Discussion}

\subsection{Study 1 - Health Benefits to the Hamsters}

A trial test was carried out to assess the benefits of the system through regular exercise to the hamsters. All hamsters had their mean Body Condition Scoring (BCS) 9] taken at the first week of the experiment. For six weeks, each hamster is given one spoon of food daily [5]. The hamsters were allowed to play Metazoa Ludens for an hour each on every weekday for the period of six weeks. At the end of the sixth week their mean BCSs were taken again. The results are shown in Figure 2 .

By using Wilcoxon signed-rank test, Metazoa Ludens was found to be able to change the BCS of the subject hamsters over the study period $(z=-3.8230$, $p=0.0006$, Wilcoxon signed-rank test). Further statistical analysis of the mean BCS of the hamsters in the 6th week using Wilcoxon signed-rank test showed that the mean BCS of hamsters after 6 weeks of using Metazoa Ludens tend towards $3(z=-1.4154, p=0.1586$, Wilcoxon signed-rank test), which is the optimal BCS score for hamsters. Hence it can be concluded that after 6 weeks of playing with Metazoa Ludens, the hamsters are getting healthier and their body condition tends to optimal. Further examinations are currently being carried out with a control group, to ensure that being in a large area was not the reason for the optimal BCS scores.

\subsection{Study 2 - Hamsters' Positive Motivation to Play the Game}

Besides studying the health benefits of hamsters, a separate study was carried out to measure the motivation of the hamsters to play Metazoa Ludens. In this study

Study 1

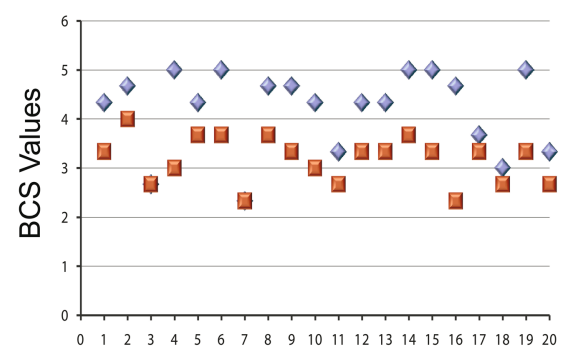

Hamster

BCS in 1st Week $\quad$ BCS in 6th Week
Study 2

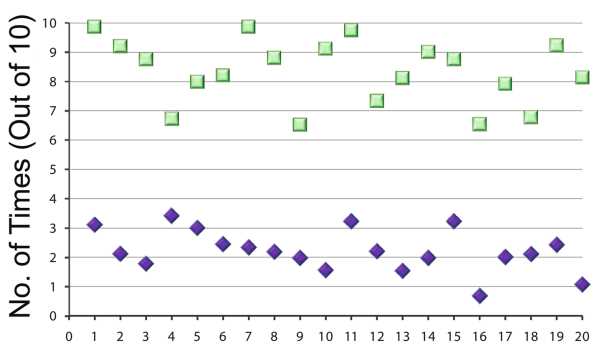

Hamster

1st Week $\square$ 4th Week

Fig. 2. Statistical results for studies 1 and 2 
the method of Duncan [20] was adapted to assess the strength of preference of the hamsters towards Metazoa Ludens. The study was carried out for four weeks and the mean percentage for the number of times each hamster chose to play Metazoa Ludens in the 1st week was compared to that in the fourth week. It was shown that the mean number of times taken for the hamsters to play the game per day increased over the study period. Further statistical analysis showed that this increase was a $60 \%$ increment. As it is shown that the hamsters increasingly chooses to play Metazoa Ludens during the study period, we conclude that the hamsters have a positive desire to play Metazoa Ludens.

The results are given in Figure 2. By using the single-tailed Wilcoxon signedrank test, it was shown that the mean number of times taken for the hamsters to play the game per day increased over the study period $(z=3.9199, p$ equal to 1.0000, Wilcoxon signed-rank test). With the two-tailed Wilcoxon signed-rank test it was shown that this increase was by 6 times per day out of the 10 possible given chances ( $z=0.7467, p=1.5468$, Wilcoxon signed-rank test), that is $60 \%$ increment. Hence based on study from Study 2 it may be concluded that with time, the number of times the hamsters chose to play the game of Metazoa Ludens increases even with the presence of a minor obstacle (as presented in the form of an additional tunnel). Based on the Method-of-Duncan, it could thus be shown that the hamsters are willing to play the game on its own free will.

\subsection{Study 3 - Users' Enjoyment Based on Flow}

For the users, a user survey was carried out to evaluate Metazoa Ludens system as a game. Game flow of the system was then broken down using features as described by Csikszentmihalyi's Flow theory [1] to evaluate the users' enjoyment of the game. Metazoa Ludens game flow is broken down as follows:

- Concentration: Concentration on the game is needed and players should be able to concentrate on the game.

- Challenge: The game should contain challenges that match the players' skill level.

- Player Skills: Player skill development and mastery should be supported in the game.

- Control: Player should be able to feel in control of their actions in the game.

- Clear Goals: Clear defined goals should be given to the players at appropriate times.

- Feedback: Appropriate feedback from the game at appropriate times is given.

- Connection: Players should feel deeply connected to the game and with little/no effort.

- Social Interaction: The game should support social interaction as well as create opportunities for it.

The original set of questions for the Flow model has been created for a generic task (like surfing the Internet) therefore some questions have been modified to 
Table 1. User Evaluation Questions based on Flow model

\begin{tabular}{|c|c|}
\hline Element & Question \\
\hline \multirow[t]{2}{*}{ Concentration } & 1) Did the game grab your attention and maintain your focus? \\
\hline & 2) Can you concentrate on the tasks at hand in the game? \\
\hline \multirow[t]{3}{*}{ Challenge } & 3) Does the game skills needed match yours? \\
\hline & 4) Do you think the game provide different levels of challenge for different players? \\
\hline & 5) As the game progresses does it become more challenging? \\
\hline \multirow[t]{2}{*}{ Player Skills } & 6) Are you able to play the game without spending too much time at the instructions? \\
\hline & 7) Is learning how to play the game fun? \\
\hline \multirow[t]{2}{*}{ Control } & 8) Do you feel in control of your character in the game? \\
\hline & 9) Do you feel in control of the game shell (starting, stopping, saving etc.)? \\
\hline \multirow[t]{2}{*}{ Clear Goals } & 10) Is the objective of the game clear and presented early? \\
\hline & 11) Are intermediate game goals clear and presented at appropriate times? \\
\hline \multirow[t]{3}{*}{ Feedback } & 12) Do you have a clear picture of your progress to the game goals at any point of time? \\
\hline & 13) Does the game give you immediate feedback of your actions? \\
\hline & 14) Do you always know your health points and time remaining? \\
\hline \multirow[t]{3}{*}{ Connection } & 15) During gameplay are you less aware of what is happening physically around you? \\
\hline & 16) Are you aware of the passing time during gameplay? \\
\hline & 17) Do you feel emotionally involved in the game? \\
\hline \multirow[t]{2}{*}{ Social Interaction } & 18) Do you feel the competition against the pets and other players (if any)? \\
\hline & $\begin{array}{l}\text { 19) Does the game support social communities (for both human players and pets) } \\
\text { inside and outside the game? }\end{array}$ \\
\hline \multirow[t]{2}{*}{ Human-animal interaction } & 20) Are you aware that you are playing with a hamster during gameplay? \\
\hline & 21) Do you feel more connected to the pets after gameplay? \\
\hline
\end{tabular}

adapt to the Metazoa Ludens environment. Questions related to human-animal interaction are added as well. Table 1 illustrates the questions and the corresponding criterion and elements in the Flow model.

Subjects: 20 subjects from a local group of hamster lovers community were randomly selected with an average age of 25.4 years old (55\% male and $45 \%$ female). Data collected from the survey after game play are expressed as mean and standard deviation unless otherwise specified. Results of the survey are given in Table 2, Of all elements explored with this survey, most of them performed positively in the survey as more than $50 \%$ selected the favorable choice to the questions posed. Nevertheless it is noted that the element Social Interaction did not score as well as the rest.

For Social Interaction, the majority agreed that they do feel the presence of social interaction at work, considering that the players are new to the game and having to cope with getting use to the controls and the game play in realtime, should be considered a positive result. A possible way to improve Social Interaction further would be to include video and voice feature which will then allow players to "see" and "speak" to the hamsters over the Internet as the hamsters may be able to recognize their owners' voice. 
Table 2. Results of Survey

\begin{tabular}{|r|r|r|r|r|r|}
\hline \multirow{2}{*}{ Qn } & \multicolumn{5}{|c|}{ Options } \\
\cline { 2 - 6 } & Yes, very & Yes & Fairly & Not really & No \\
\hline 1 & $75.00 \%$ & $25.00 \%$ & $0.00 \%$ & $0.00 \%$ & $0.00 \%$ \\
\hline 2 & $70.00 \%$ & $10.00 \%$ & $20.00 \%$ & $0.00 \%$ & $0.00 \%$ \\
\hline 3 & $65.00 \%$ & $20.00 \%$ & $15.00 \%$ & $0.00 \%$ & $0.00 \%$ \\
\hline 4 & $70.00 \%$ & $30.00 \%$ & $0.00 \%$ & $0.00 \%$ & $0.00 \%$ \\
\hline 5 & $60.00 \%$ & $20.00 \%$ & $20.00 \%$ & $0.00 \%$ & $0.00 \%$ \\
\hline 6 & $95.00 \%$ & $5.00 \%$ & $0.00 \%$ & $0.00 \%$ & $0.00 \%$ \\
\hline 7 & $85.00 \%$ & $15.00 \%$ & $0.00 \%$ & $0.00 \%$ & $0.00 \%$ \\
\hline 8 & $95.00 \%$ & $5.00 \%$ & $0.00 \%$ & $0.00 \%$ & $0.00 \%$ \\
\hline 9 & $65.00 \%$ & $20.00 \%$ & $10.00 \%$ & $5.00 \%$ & $0.00 \%$ \\
\hline 10 & $95.00 \%$ & $5.00 \%$ & $0.00 \%$ & $0.00 \%$ & $0.00 \%$ \\
\hline 11 & $70.00 \%$ & $5.00 \%$ & $20.00 \%$ & $5.00 \%$ & $0.00 \%$ \\
\hline 12 & $65.00 \%$ & $15.00 \%$ & $20.00 \%$ & $0.00 \%$ & $0.00 \%$ \\
\hline 13 & $65.00 \%$ & $15.00 \%$ & $15.00 \%$ & $5.00 \%$ & $0.00 \%$ \\
\hline 14 & $85.00 \%$ & $5.00 \%$ & $10.00 \%$ & $0.00 \%$ & $0.00 \%$ \\
\hline 15 & $95.00 \%$ & $5.00 \%$ & $0.00 \%$ & $0.00 \%$ & $0.00 \%$ \\
\hline 16 & $60.00 \%$ & $40.00 \%$ & $0.00 \%$ & $0.00 \%$ & $0.00 \%$ \\
\hline 17 & $40.00 \%$ & $50.00 \%$ & $10.00 \%$ & $0.00 \%$ & $0.00 \%$ \\
\hline 18 & $15.00 \%$ & $70.00 \%$ & $10.00 \%$ & $5.00 \%$ & $0.00 \%$ \\
\hline 19 & $15.00 \%$ & $55.00 \%$ & $20.00 \%$ & $10.00 \%$ & $0.00 \%$ \\
\hline 20 & $60.00 \%$ & $10.00 \%$ & $15.00 \%$ & $15.00 \%$ & $0.00 \%$ \\
\hline 21 & $65.00 \%$ & $30.00 \%$ & $5.00 \%$ & $0.00 \%$ & $0.00 \%$ \\
\hline
\end{tabular}

\section{$5 \quad$ Framework for Human-Animal Interaction System}

A framework for describing human-animal interaction system and the interactions involved may be developed from the built of Metazoa Ludens. This can provide guidelines for future human-animal interaction systems to be based on, as well as provide possible insights for new human-animal interaction systems. Five design dimensions for human-animal interaction system are presented. Existing human-animal interaction systems are ranked upon these five dimensions. The dimensions are:

- Habitat design: The area of interaction should be safe, comfortable and suitable for the animal. Choices available for this dimension are - Native, where the environment used is where the animal normally resides; Recreated, where the environment is not where the animal normally can be found but yet not unsuitable for the animal to stay in; Unsuitable, where the environment is not suitable for the animal.

- Ease of use: The way to interact with the system should come naturally to the animal since it is not an easy task to teach the animal to for example use a keyboard or mouse as input. The natural behavior of animals thus needs to be studied and modeled. Choices available for this dimension are Instinctive, where the actions required from the animal is instinctive to what the animal normally do; Learnt, where the animal requires certain learning process to perform the tasks required; Unsuitable, where the actions required are unsuitable to be performed by the animal.

- Interactivity: System should allow interaction between pet and owner via the human-animal interactive system. Choices available for this dimension are - None, where no interactivity is given between human and the animal; One-way where interaction is only one-way; Two-way where interaction is two-ways.

- Pet's choice: While the system allows users to play with their pets, such human-animal interactive system should consider giving the animals a choice 

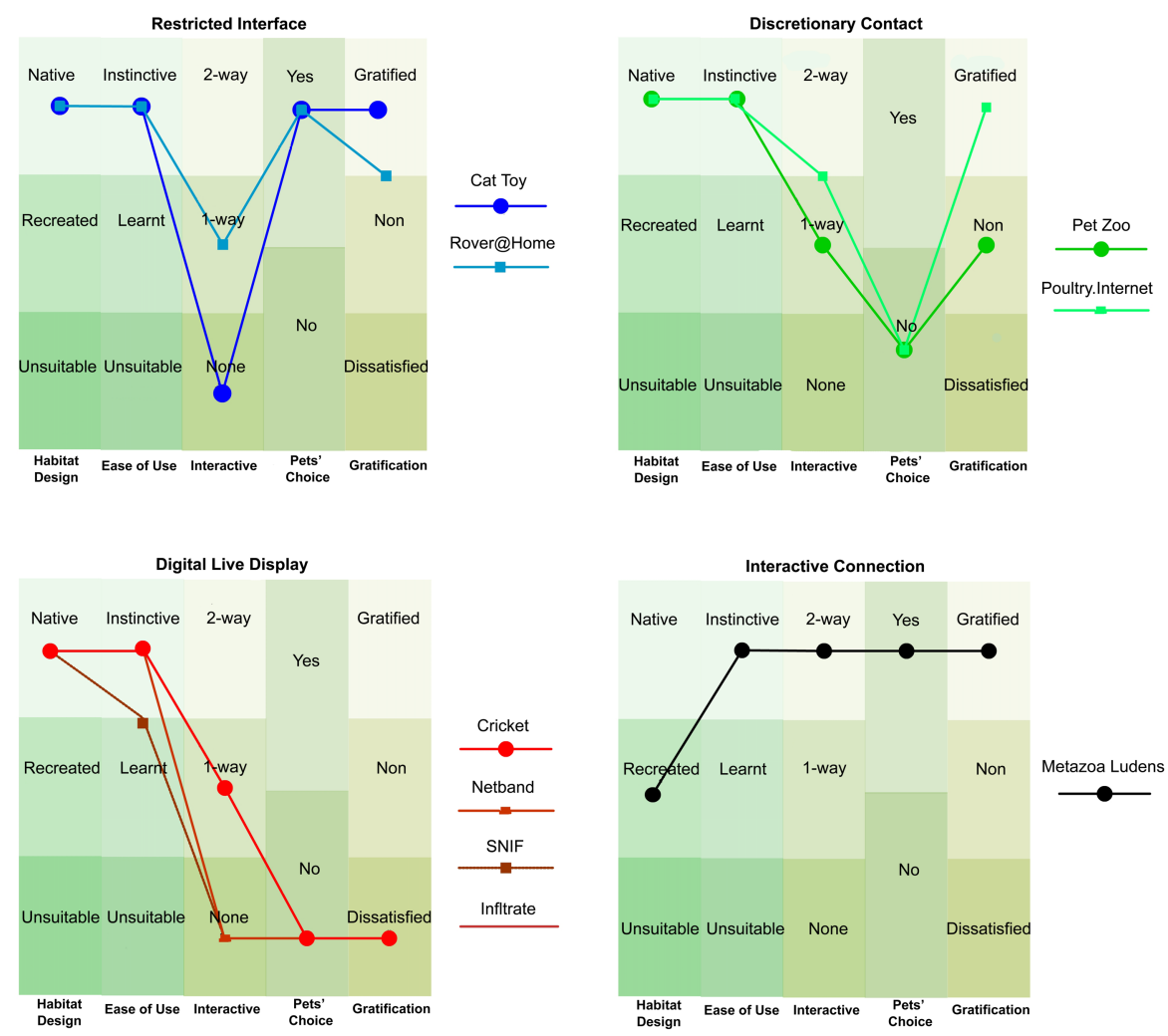

Fig. 3. Design Pattern for Human-Animal Interaction System

for interactivity. Choices available for this dimension are - Yes, where animals are given the choice to use the system; No, where animals are not given the choice to use the system.

- Animal's Gratification: There should be some form of gratification (be it health benefits or entertainment) for the animal in using the system, else it would be just a case of making the animal perform certain tasks for the entertainment of the human. Choices available for this dimension are - Gratified, where the animals are given some benefits or gratification from using the system; No, where animals are given gratification nor given dissatisfaction from using the system; Dissatisfied, where animals gets negative gratification from using the system.

Based on the clustering of systems, four design patterns for human-animal interaction system (after pattern language for architectural studies [21]) are obtained, see Figure 3. They are: Restricted Interface accounts for systems like Cat Toy. Such systems score fairly well in four of the dimensions except for Interactivity. Interaction is mostly one-way, from human to animal; the animal has no means to interact directly with the human. Such systems are mostly intended 
to have a restricted one-way interface focusing on the human's interaction with the animal. Discretionary Contact accounts for systems like Poultry.Internet and Pet Zoo, and are mostly intended for the human to interact with the animal at the human's discretion. Despite the animals having little choice in participating in this interaction, it is noted that gratification for the animal is high. Digital Live Display accounts for systems like Infiltrate. Such systems score very well in Habitual Design and Ease of Use while scoring very low for the remaining dimensions. These systems generally focus on being a means of digital live display for the human with little emphasis on the animal's choice to interact, their interaction with the human and their gratification from such systems. Interactive Connection accounts for systems like Metazoa Ludens. This archetype scores very well in all dimensions except for Habitual Design. This archetype focuses on bidirectional interaction as well as the animal's choice to play and its gratification, it also ensures the ease of use of the system by the animal. Such systems are mostly intended for equal emphasis on interaction between the human and the animal for connection. While the four presented system design can help designers in future design as model for development of new human-animal interaction systems, it does not represent the only possibilities for building such systems. More importantly will be the use of the five dimensions in consideration of the new design. In an ideal situation any such systems to be built should be high in all five dimensions.

\section{Conclusion}

Metazoa Ludens presents a mixed reality game which allows gameplay between humans and small animals (like hamsters) over the Internet. This system not only allows a novel game play but allows humans and hamsters to interact meaningfully and effectively with a mixed reality system. This type of interaction offered gives the enrichment and enhancement of the experience as brought about by digitalized system. While not trying to replace conventional interaction between humans and small animals, its aim is to offer remote interaction with the small animals as well as another way of interacting with the small animals through advanced digital technology. We not only showed detailed experimental verification and positive results, but deeply aimed this work to provide lessons and framworks for future human-animal media designs for the benefit of animal and human relations.

Acknowledgments. We would like to thank all members of Mixed Reality Lab, Singapore for all their help rendered.

\section{References}

1. Csikszentmihalyi, M.: Beyond Boredom and Anxiety: Experiencing Flow in Work and Play. Jossey-Bass Inc., San Francisco, California (1975)

2. Kawakami, N., Haratani, T.: Epidemiology of Job Stress and Health in Japan: Review of Current Evidence and Future Direction. Industrial Health 37, 174-186 (1999) 
3. Teh, K.S.J., Lee, S.P., Cheok, A.D.: Novel Uses of Existing Wearable Sensors Technology. In: ISWC, vol. 1(2), pp. 18-21. IEEE Press, Los Alamitos (2005)

4. Phaosavasdi, S., Thaneepanichskul, S., Tannirandorn, Y., Thamkhantho, M., Pruksapong, C., Kanjanapitak, A., Leong, H.: Journal of Medical Association of Thailand 88, 287-293 (2005)

5. Zim, H.S.: Golden Hamster. Morrow, New York (1951)

6. Pet Web Site (2007), Available Online http://www.petwebsite.com/

7. National Hamster Council (2007), Available Online http://www.hamster-uk.org

8. Hamster Land (2007), Available Online http://www.hamster.com/

9. Ullman-Cullere, M.H., Foltz, C.J.: Body Condition Scoring: A Rapid and Accurate Method for Assessing Health Status in Rodents. Laboratory Animal Science 49, 319-323 (1999)

10. Sweetser, P., Wyeth, P.: GameFlow: A Model for Evaluating Player Enjoyment in Games. Computers in Entertainment (CIE) 3(3), 14-27 (2005)

11. Resner, B.: Rover@home. MIT, Cambridge, Massachusetts, USA (2001)

12. CME (1999), Available Online from http://www.metahuman.org/web/pzoo1.html/

13. Mikesell, D.: Networking Pets and People. In: Adjunct Proceedings of Ubicomp, Seattle, USA, pp. 88-89 (2003)

14. Goldberg, K., Perona, P., Bender, J., Lobel, I., Chen, K.: On Exhibit: Neuro. Caltech336. Pasadena, CA, USA (2003)

15. Gips, J., Fields, N., Liang, P., Pilpre, A.: SNIF: Social Networking in Fur. In: CHI '05: CHI '05 extended abstracts on Human factors in computing systems, vol. 2(7), pp. 1391-1394. ACM Press, New York (2005)

16. Netband (1994), Available Online http://www.buitenland.org/

17. Behrens, D.: Keeping up with the Tamagotchis/ A Report on Virtual Pets and Knockoff Reality. Newsday, 6 (1997)

18. Cheok, A.D., Goh, K.H., Liu, W., Fabiz, F., Fong, S.W., Teo, S.L., Li, Y., Yang, $\mathrm{X}$.: Human Pacman: a mobile, wide-area entertainment system based on physical, social, and ubiquitous computing. Personal and Ubiquitous Computing 8(2), 71-81 (2004)

19. Tan, K.C.R., Cheok, A.D., Teh, K.S.: Metazoa Ludens: Mixed Reality Environment for Playing Computer Games with Pets. The International Journal of Virtual Reality 5(3), 53-58 (2006)

20. Duncan, I.J.H., Kite, V.G.: Some investigations into motivation in the domestic fowl. Applied Animal Behavior Science 18, 387-388 (1987)

21. Alexander, C.: A Pattern Language: Towns, Buildings, Construction. Oxford University Press, Oxford (1977) 